\title{
Comparison of serum fibrosis biomarkers for diagnosing significant liver fibrosis in patients with chronic hepatitis B
}

\author{
YUKI TSUJI, TADASHI NAMISAKI, KOSUKE KAJI, HIROAKI TAKAYA, KEISUKE NAKANISHI, \\ SHINYA SATO, SOICHIRO SAIKAWA, YASUHIKO SAWADA, KOU KITAGAWA, NAOTAKA SHIMOZATO, \\ HIDETO KAWARATANI, KEI MORIYA, RYUICHI NOGUCHI, TAKEMI AKAHANE, \\ AKIRA MITORO and HITOSHI YOSHIJI
}

Third Department of Internal Medicine, Nara Medical University, Kashihara, Nara 634-8522, Japan

Received June 24, 2019; Accepted February 04, 2020

DOI: $10.3892 / \mathrm{etm} .2020 .8798$

\begin{abstract}
Chronic hepatitis B (CHB) virus continues to be a leading cause of morbidity and mortality worldwide. The diagnosis of liver fibrosis has a key role in selecting patients with CHB for antiviral treatment. However, serum biomarkers demonstrate limited diagnostic utility. The present study aimed to compare the performances of fibrosis biomarkers for diagnosing significant liver fibrosis that indicates the need for antiviral therapy in patients with $\mathrm{CHB}$ and to identify the most appropriate biomarker for these patients. The current study included 96 antiviral-naïve patients with CHB who underwent liver biopsy. METAVIR scoring system was used to assess liver fibrosis and necroinflammation. The diagnostic performances were evaluated of the platelet (PLT) count; the levels of hyaluronan, serum 7S domain of type 4 collagen, procollagen type III N-terminal peptide, tissue inhibitor of metalloproteinases 1, Mac-2 binding protein glycosylation isomer (M2BPGi) and $\mathrm{N}$-terminal type III collagen propeptide (Pro-C3); the fibrosis index based on four factors; the aspartate aminotransferase-to-platelet ratio index; and enhanced liver fibrosis score for identifying significant liver fibrosis $[\geq$ fibrosis stage 2 (F2)]. All fibrosis biomarkers, except the Pro-C3 level,
\end{abstract}

Correspondence to: Dr Tadashi Namisaki, Third Department of Internal Medicine, Nara Medical University, 840 Shijo-cho, Kashihara, Nara 634-8522, Japan

E-mail: tadashin@naramed-u.ac.jp

Abbreviations: 7S collagen, 7S domain of type 4 collagen; ALT, alanine aminotransferase; APRI, aspartate aminotransferase-toplatelet ratio index; AST, aspartate aminotransferase; $\mathrm{CHB}$, chronic hepatitis B; ELF, enhanced liver fibrosis score; FIB-4, fibrosis index based on four factors; HA, hyaluronan; M2BPGi, mac-2-binding protein glycosylation isomer; PIIINP, type 3 procollagen-N-peptide; Pro-C3, N-terminal type III collagen propeptide; TIMP-1, tissue inhibitor of metalloproteinase 1

Key words: chronic hepatitis B, liver fibrosis, liver necroinflammation, Mac-2 binding protein glycosylation isomer, $\mathrm{N}$-terminal type III collagen propeptide correlated with the fibrosis stage. M2BPGi was better than other biomarkers for diagnosing $\geq F 2$, with the highest area under the curve of 0.902 . M2BPGi demonstrated a higher diagnostic accuracy for significant fibrosis than mild/severe fibrosis or cirrhosis. However, no significant correlation was observed between the M2BPGi level and fibrosis stage in patients with $\mathrm{CHB}$ having significant liver necroinflammation defined as $\geq$ necroinflammatory activity 2 . The M2BPGi level and PLT count were exclusively correlated with the fibrosis stage in 73 patients without significant liver necroinflammation. M2BPGi demonstrated the highest diagnostic performance for significant fibrosis in patients having significant liver fibrosis with no significant liver necroinflammation. In conclusion, the M2BPGi level can accurately diagnose significant liver fibrosis that indicates the need for antiviral therapy in patients with CHB.

\section{Introduction}

Liver fibrosis is a common pathological manifestation of various chronic liver diseases, which can advance to cirrhosis and hepatocellular carcinoma (1) Liver biopsy is the gold standard approach to assess fibrosis progression in chronic liver diseases, such as chronic hepatitis B (CHB), chronic hepatitis $\mathrm{C}(\mathrm{CHC})$, autoimmune liver diseases and non-alcoholic fatty liver disease, even though it is an invasive procedure (2). As the progression of liver fibrosis can be reversed with treatment, serial hepatic biopsy analysis is important for chronic liver diseases (3). However, repetitive procedures are difficult to perform because of various limitations regarding the principle, cost and sampling error (3). Therefore, non-invasive methods for assessing liver fibrosis are required to overcome these limitations. Non-invasive techniques, such as magnetic resonance imaging (MRI) (4) and ultrasound-based transient elastography, are considered helpful for estimating the advanced stages of fibrosis (5). Serum surrogate biomarkers can also be employed and they are broadly classified as direct markers, which reflect alterations in the contents of extracellular matrix proteins, and indirect markers, which reflect changes in liver function. Direct markers include hyaluronan (HA), 7S domain of type 4 collagen (7S collagen) and procollagen type III N-terminal peptide (PIIINP), and with all these markers, there are 
difficulties in discriminating accurately between the early and adjacent stages of fibrosis $(6,7)$. Conversely, indirect markers involve routine laboratory parameters and are calculated from these laboratory parameters [for example, fibrosis index based on four factors (FIB-4 index) and aspartate aminotransferase (AST)-to-platelet ratio index (APRI)] (8). These are inefficient in detecting early stage fibrosis in patients with CHB (9).

The early diagnosis of liver fibrosis is important to control disease progression. The clinical practice guidelines for the management of hepatitis B proposed by the American Association for the Study of Liver Diseases (10) and the Japan Society of Hepatology (11) state that the decision to initiate antiviral therapy should be taken if a patient has F1 fibrosis without necroinflammation. The underlying mechanisms of histological progression vary among patients with chronic liver diseases $(12,13)$. Recently, plasma N-terminal type III collagen propeptide (Pro-C3) has been introduced as a novel non-invasive marker for the assessment of liver fibrosis in patients with CHC (14) and non-alcoholic steatohepatitis (15). The authors of the present study have previously demonstrated that the serum angiotensin-converting enzyme level is a beneficial non-invasive marker for evaluating significant fibrosis in patients with $\mathrm{CHB}$ without fatty liver or habitual alcoholic consumption (16). To date, no surrogate marker that accurately quantifies hepatic fibrosis has been identified in patients with $\mathrm{CHB}$. The present study aimed to compare the performances of fibrosis biomarkers for diagnosing significant liver fibrosis that indicate the need for antiviral therapy in patients with $\mathrm{CHB}$ to identify the most appropriate biomarker in these patients.

\section{Materials and methods}

Patients. The present study enrolled 96 treatment-naïve patients who were diagnosed with CHB serologically and histologically between September 2005 and May 2017 (Table I). The typical characteristics of CHB infection were as follows: i) Hepatitis B surface antigen ( $\mathrm{HBsAg}$ ) positivity for at least 6 months; and ii) serum hepatitis B virus (HBV) DNA level $\geq 1.3 \log$ $\mathrm{IU} / \mathrm{ml}$. Detections of HB envelope antigen, anti-HBe $\mathrm{IgG}$ and anti-HB core IgG were not considered as inclusion criteria in the present assessment. The exclusion criteria were clinical findings suggestive of concomitant liver diseases (including $\mathrm{CHC}$ ), autoimmune hepatitis, primary biliary cholangitis, alcoholic liver disease, non-alcoholic fatty liver disease and hepatocellular carcinoma. The present study was performed in accordance with the standards of the Declaration of Helsinki and written informed consent was obtained from all the study participants. The Ethics Committee of Nara Medical University affiliated Hospital approved this study (approval no. 1077).

Laboratory analysis and measurement of clinical laboratory parameters. Variables, including age, platelet (PLT) count and levels of AST, alanine aminotransferase (ALT), albumin (ALB), total bilirubin, HBV DNA, and HBsAg, were assessed and recorded on admission (Table I). Additionally, the HA level, $7 \mathrm{~S}$ collagen level, PIIINP level, tissue inhibitor of metalloproteinases 1 (TIMP-1) level, Mac-2 binding protein glycosylation isomer (M2BPGi) level, Pro-C3 level, FIB-4 index, APRI and enhanced liver fibrosis (ELF) score were used as non-invasive biomarkers for the assessment of liver fibrosis. The following formulas were used: FIB-4 index $=($ age $\mathrm{x}$ AST $) /[(\mathrm{PLT}$ count $) \mathrm{x}$ (ALT)1/2]; APRI=[(sample AST/reference AST) x100]/PLT count ELF score $=2.278+[0.851 \ln (\mathrm{HA})+0.751 \ln (\mathrm{PIIINP})+$ $0.394 \ln$ (TIMP-1)]. The levels of HA, TIMP-1 and PIIINP were measured using chemiluminometric immunoassays performed on the ADVIA Centaur XP Immunoassay System (Siemens Healthineers) $(17,18)$. Serum $7 \mathrm{~S}$ collagen was determined using radioimmunoassay kits (7S-RIA; Nippon DPC Corporation) (19). The Wisteria floribunda agglutinin-positive Mac-2 binding protein assay was performed using an automated chemiluminescence enzyme immunoassay analyzer (HISCL-5000; Sysmex Corporation) (20). Pro-C3 level was measured using UniQ PIIINP RIA assay (Orion Diagnostica Ltd.) (21). HBs antigen and HBV DNA levels were measured as previously described (22).

Liver biopsy. Percutaneous liver biopsy was performed before the initiation of therapy, using ultrasound localization. Liver samples were fixed in formalin at a room temperature of $20-22^{\circ} \mathrm{C}$, embedded in paraffin and sectioned to $5 \mu \mathrm{m}$. Each section was stained with hematoxylin-eosin and reticular fiber stain for $30 \mathrm{sec}$ at a room temperature of 20 to $22^{\circ} \mathrm{C}$ or Masson's stain for $60 \mathrm{~min}$ at $54-64^{\circ} \mathrm{C}$. Professor Chiho Obayashi and Dr Kohei Morita (Department of Diagnostic Pathology, Nara Medical University) independently reviewed all cases for validation of the histological features of $\mathrm{CHB}$. The METAVIR scoring system (23) was used to evaluate fibrosis and necroinflammation. The degree of hepatic fibrosis was scored from F0 to F4 (F0, no fibrosis; F1, portal fibrosis without septa; F2, portal fibrosis with few septa; F3, numerous septa without cirrhosis; and F4, cirrhosis) $(11,24)$. The degree of necroinflammatory activity was scored from A0 to A3 (A0, no histological necroinflammatory activity; A1, minimal necroinflammatory activity; A2, moderate necroinflammatory activity; and A3, severe necroinflammatory activity) (25). F0-F1 and A0-A1 were considered to indicate no to mild fibrosis and no to mild necroinflammation, whereas F2-F4 and A2-A3 were considered to indicate moderate to severe fibrosis and cirrhosis and moderate to severe necroinflammation, respectively. Significant liver fibrosis and necroinflammation were defined as the fibrosis stage $\geq \mathrm{F} 2$ and necroinflammation grade $\geq \mathrm{A} 2$, respectively.

Statistical analysis. Patient characteristics are presented as the mean \pm standard error of the mean. Differences in continuous variables were assessed using Student's t-tests or one-way ANOVAs followed by Tukey's post-hoc tests. The Mann-Whitney U test was used to compare two groups of nonparametric data. Chi-square test was used to analyze categorical variables. Correlations were evaluated using Spearman's correlation coefficient for continuous variables. All statistical tests were two-tailed and $\mathrm{P}<0.05$ was considered to indicate a statistically significant difference. The areas under the receiver operating characteristic (ROC) curves (AUCs) were used to evaluate the diagnostic values of the fibrosis biomarkers with regard to the correct identification of significant liver fibrosis. The sensitivities, specificities, positive-predictive values (PPVs), negative-predictive values 
(NPVs), diagnostic accuracies and cut-off values of the fibrosis biomarkers were calculated from the ROC curves. All analyses were performed using SPSS software version 24 (IBM Corp.).

\section{Results}

Baseline clinical characteristics of patients with different fibrosis stages. The demographic and baseline characteristics of the patients are summarized in Table I. The fibrosis stages were F0, F1, F2, F3 and F4 in 25 (26.0\%), 44 (45.8\%), $14(14.6 \%), 10(10.4 \%)$ and $3(3.2 \%)$ patients, respectively (Table II). Spearman's rank correlation coefficients between the fibrosis stage and PLT count, HA level, 7S collagen level, PIIINP level, TIMP-1 level, M2BPGi level, Pro-C3 level, FIB-4 index, APRI and ELF score were -0.43, 0.38, 0.52, 0.54, 0.38, $0.61,0.08,0.42,0.58$ and 0.55 , respectively (Fig. 1A-J). All fibrosis biomarkers, except the Pro-C3 level, were significantly associated with the liver fibrosis stage in patients with $\mathrm{CHB}$ (Table II).

Levels of serum fibrosis biomarkers according to the degree of liver fibrosis in patients with $\mathrm{CHB}$. It was identified that the M2BPGi level was markedly higher in patients with F2 fibrosis compared with those with F1 fibrosis [F1, 0.75 \pm 0.45 vs. F2, 1.29 \pm 0.46 Cutoff index (COI) $\mathrm{P}<0.01$; Fig. 2F]. Unlike the M2BPGi findings, there were significant differences between patients with F2 fibrosis and those with F3 fibrosis with regard to the HA level (F2, 53.4 \pm 23.6 vs. F3, $245.9 \pm 278.9 \mathrm{ng} / \mathrm{ml}$; $\mathrm{P}<0.01$; Fig. $2 \mathrm{~B}), 7 \mathrm{~S}$ collagen level $(\mathrm{F} 2$, $4.0 \pm 0.9$ vs. F3, $6.9 \pm 2.7 \mathrm{ng} / \mathrm{ml}$; $\mathrm{P}<0.01$; Fig. 2 C), PIIINP level (F2, 11.4 \pm 4.2 vs. F3, 17.3 $\pm 7.2 \mathrm{ng} / \mathrm{ml}$; P<0.05; Fig. 2D), APRI (F2, $0.77 \pm 0.34$ vs. F3, 1.52 \pm 1.03 ; $\mathrm{P}<0.01$; Fig. 2I) and ELF score (F2, 9.51 \pm 0.68 vs. F3, 10.79 \pm 1.15 ; P<0.01; Fig. $2 J)$ and between patients with F3 fibrosis and those with F4 fibrosis with regard to the FIB-4 index (F3, 2.71 \pm 1.62 vs. F4, 4.97 \pm 3.02 ; $\mathrm{P}<0.05$; Fig. $2 \mathrm{H}$ ). However, no differences were found among the fibrosis groups with regard to the PLT count and serum TIMP-1 and Pro-C3 levels (Fig. 2A, E and G).

Diagnostic performances of serum fibrosis biomarkers for identifying significant liver fibrosis in patients with $\mathrm{CHB}$. The diagnostic sensitivity, specificity, PPV, NPV and accuracy of the PLT count, HA level, 7S collagen level, PIIINP level, TIMP-1 level, M2BPGi level, Pro-C3 level, FIB-4 index, APRI and ELF score for the differentiation of $\geq \mathrm{F} 2$ fibrosis in patients with CHB are shown in Table III. The AUCs of these markers for the accurate diagnosis of significant liver fibrosis $(\geq \mathrm{F} 2)$ were $0.757,0.776,0.739,0.778,0.713,0.902,0.452$, $0.676,0.812$ and 0.816 , respectively (Table III). These findings indicated that the serum M2BPGi level was most accurately identifying significant liver fibrosis when compared with other non-invasive fibrosis biomarkers in patients with $\mathrm{CHB}$.

Diagnostic performance of M2BPGi for identifying the different stages of liver fibrosis in patients with $C H B$. The AUCs for identifying mild fibrosis (F1-4), significant fibrosis (F2-4), severe (F3-4) and advanced fibrosis/cirrhosis (F4) were 0.773 (sensitivity, 52.1\%; specificity, 100\%; Fig. 3A) 0.902 (sensitivity, 92.6\%; specificity, 82.4\%; Fig. 3B), 0.865 (sensitivity, 100\%; specificity, 63.4\%; Fig. 3C) and 0.774 (sensitivity,
Table I. Baseline characteristics of patients with CHB.

Variable

CHB patients $(n=96)$

Sex

Male

49

Female

47

Age, years

$51.1 \pm 13.7$

Fibrosis stage

F0

25

F1

44

F2

14

F3

10

F4

3

Inflammatory activity

A0

36

A1

37

A2

20

A3

3

Platelet $\left(10^{4} / \mu 1\right)$

$19.7 \pm 5.4$

AST (IU/l)

$37.7 \pm 27.2$

ALT (IU/l)

$46.8 \pm 57.3$

Serum Albumin $(\mathrm{g} / \mathrm{dl})$

$4.2 \pm 0.3$

Total Bilirubin (mg/dl)

$0.9 \pm 0.3$

HBV DNA (Log copies/ml)

$4.8 \pm 2.6$

HBs Antigen (IU/ml)

Hyaluronic acid (ng/ml)

$13,782 \pm 30,650$

$83.7 \pm 15.0$

$4.3 \pm 2.2$

Type 4 collagen $7 \mathrm{~S}(\mathrm{ng} / \mathrm{ml})$

$10.4 \pm 6.4$

PIIINP (ng/ml)

$224.9 \pm 73.8$

TIMP-1 (ng/ml)

$0.87 \pm 0.51$

M2BPGi (cutoff index)

$16.6 \pm 5.2$

Pro C3 (ng/ml)

$1.77 \pm 1.31$

$0.71 \pm 0.73$

APRI

$9.34 \pm 1.10$

CHB, chronic hepatitis type B; AST, aspartate aminotransferase; ALT, alanine aminotransferase; PIIINP, type 3 procollagen-N-peptide; TIMP-1, tissue inhibitor of metalloproteinase 1; M2BPGi, Mac-2-binding protein glycosylation isomer; Pro-C3, N-terminal type III collagen propeptide; FIB-4, fibrosis index based on four factors; APRI, the aspartate aminotransferase-to-platelet ratio index; ELF, enhanced liver fibrosis.

100\%; specificity, 56.5\%; Fig. 3D), respectively, indicating that M2BPGi has higher diagnostic accuracy for significant fibrosis than mild/severe fibrosis or cirrhosis. Together, these results suggested that serum M2BPGi had the best performance for identifying significant liver fibrosis in patients with CHB. The diagnostic accuracy of serum M2BPGi level was compared to that of other fibrosis markers including the PLT count; HA, 7S collagen, PIIINP, TIMP-1 and Pro-C3 levels; FIB-4 index; APRI; and ELF score. Significant differences were observed in the AUCs between M2BPGi level and PLT count, HA level, 7S collagen level, PIIINP level, TIMP-1 level, Pro-C3 level and FIB-4 index $(\mathrm{P}<0.05, \mathrm{P}<0.05, \mathrm{P}<0.01$, 
Table II. Baseline characteristics of patients with chronic hepatitis B stratified according to liver fibrosis stages.

\begin{tabular}{|c|c|c|c|c|c|c|}
\hline Variable (reference range) & $\mathrm{F} 0(\mathrm{n}=25)$ & $\mathrm{F} 1(\mathrm{n}=44)$ & $\mathrm{F} 2(\mathrm{n}=14)$ & $\mathrm{F} 3(\mathrm{n}=10)$ & $\mathrm{F} 4(\mathrm{n}=3)$ & $\begin{array}{l}\text { Overall } \\
\text { P-value }\end{array}$ \\
\hline Male/female & $11 / 14$ & $23 / 21$ & $7 / 7$ & $6 / 4$ & $2 / 1$ & NS \\
\hline Age (years) & $53.7 \pm 2.4$ & $50.0 \pm 2.1$ & $49.1 \pm 3.4$ & $51.9 \pm 5.4$ & $51.6 \pm 6.7$ & NS \\
\hline Platelet $\left(10^{4} / \mu 1\right)(14.0-37.9)$ & $21.6 \pm 1.1$ & $20.6 \pm 0.7$ & $18.0 \pm 1.0$ & $15.4 \pm 4.8$ & $12.0 \pm 2.9$ & $<0.01$ \\
\hline AST (IU/l) (10-40) & $26.1 \pm 2.5$ & $34.1 \pm 3.4$ & $42.9 \pm 6.0$ & $66.3 \pm 46.3$ & $67.3 \pm 15.9$ & $<0.01$ \\
\hline $\operatorname{ALT}(\mathrm{IU} / \mathrm{l})(5-45)$ & $22.0 \pm 2.3$ & $42.4 \pm 5.4$ & $65.9 \pm 19.9$ & $97.4 \pm 118.3$ & $60.7 \pm 3.9$ & $<0.01$ \\
\hline Serum albumin $(\mathrm{g} / \mathrm{dl})(3.7-5.5)$ & $4.2 \pm 0.0$ & $4.2 \pm 0.0$ & $4.1 \pm 0.0$ & $4.0 \pm 0.4$ & $3.9 \pm 0.0$ & NS \\
\hline Total Bilirubin (mg/dl) $(0.3-1.2)$ & $0.8 \pm 0.0$ & $0.8 \pm 0.0$ & $0.9 \pm 0.0$ & $1.0 \pm 0.3$ & $1.8 \pm 0.0$ & NS \\
\hline HBV DNA (Log copies/ml) & $3.5 \pm 0.4$ & $4.8 \pm 0.4$ & $5.8 \pm 0.5$ & $6.3 \pm 2.9$ & $3.0 \pm 1.4$ & 0.01 \\
\hline HBsAg (IU/ml) $(<0.05)$ & $12,128 \pm 5,088$ & $42,946 \pm 24,337$ & $11,051 \pm 5,832$ & $11,801 \pm 24,431$ & $754 \pm 340$ & NS \\
\hline Hyaluronic acid $(\mathrm{ng} / \mathrm{ml})(<50.0)$ & $61.7 \pm 24.9$ & $52.5 \pm 10.2$ & $53.4 \pm 6.3$ & $245.9 \pm 88.1^{\mathrm{b}}$ & $325.1 \pm 165.6$ & $<0.01$ \\
\hline Type 4 collagen $7 \mathrm{~S}(\mathrm{ng} / \mathrm{ml})(<6.0)$ & $3.6 \pm 0.2$ & $3.8 \pm 0.2$ & $4.0 \pm 0.2$ & $6.9 \pm 0.9^{b}$ & $9.1 \pm 3.5$ & $<0.01$ \\
\hline PIIINP (ng/ml) (3.62-9.52) & $7.9 \pm 0.4$ & $8.9 \pm 0.6$ & $11.4 \pm 1.1$ & $17.3 \pm 2.3^{\mathrm{b}}$ & $24.7 \pm 10.5$ & $<0.01$ \\
\hline TIMP-1 (ng/ml) & $198.6 \pm 13.7$ & $213.7 \pm 5.9$ & $247.9 \pm 22.1$ & $291.2 \pm 38.3$ & $277.7 \pm 27.1$ & $<0.01$ \\
\hline M2BPGi (cutoff index) $(<1.00)$ & $0.53 \pm 0.03$ & $0.75 \pm 0.07$ & $1.29 \pm 0.12^{\mathrm{a}}$ & $1.44 \pm 0.11$ & $1.42 \pm 0.38$ & $<0.01$ \\
\hline ProC3 (ng/ml) & $18.5 \pm 1.3$ & $15.5 \pm 0.5$ & $16.5 \pm 1.6$ & $18.0 \pm 1.9$ & $14.3 \pm 0.5$ & NS \\
\hline FIB-4 & $1.52 \pm 0.14$ & $1.46 \pm 0.13$ & $1.85 \pm 0.30$ & $2.71 \pm 0.51^{\mathrm{c}}$ & $4.97 \pm 1.74$ & 0.05 \\
\hline APRI & $0.43 \pm 0.03$ & $0.56 \pm 0.06$ & $0.77 \pm 0.09$ & $1.52 \pm 0.33^{\mathrm{b}}$ & $2.56 \pm 1.07$ & $<0.01$ \\
\hline ELF score & $8.91 \pm 0.18$ & $9.04 \pm 0.13$ & $9.51 \pm 0.18$ & $10.79 \pm 0.36^{\mathrm{b}}$ & $11.28 \pm 0.77$ & $<0.01$ \\
\hline
\end{tabular}

${ }^{\mathrm{a}} \mathrm{P}<0.01$ vs. F1; ${ }^{\mathrm{b}} \mathrm{P}<0.01$ vs. $\mathrm{F} 2$; ${ }^{\mathrm{C}} \mathrm{P}<0.01$ vs. F4. CHB, chronic hepatitis $\mathrm{B}$; AST, aspartate aminotransferase; ALT, alanine aminotransferase; PIIINP, type 3 procollagen-N-peptide; TIMP-1, tissue inhibitor of metalloproteinase 1; M2BPGi, Mac-2-binding protein glycosylation isomer; Pro-C3, N-terminal type III collagen propeptide; FIB-4, fibrosis index based on four factors; APRI, aspartate aminotransferase-to-platelet ratio index; ELF score, enhanced liver fibrosis score; F, fibrosis stage.
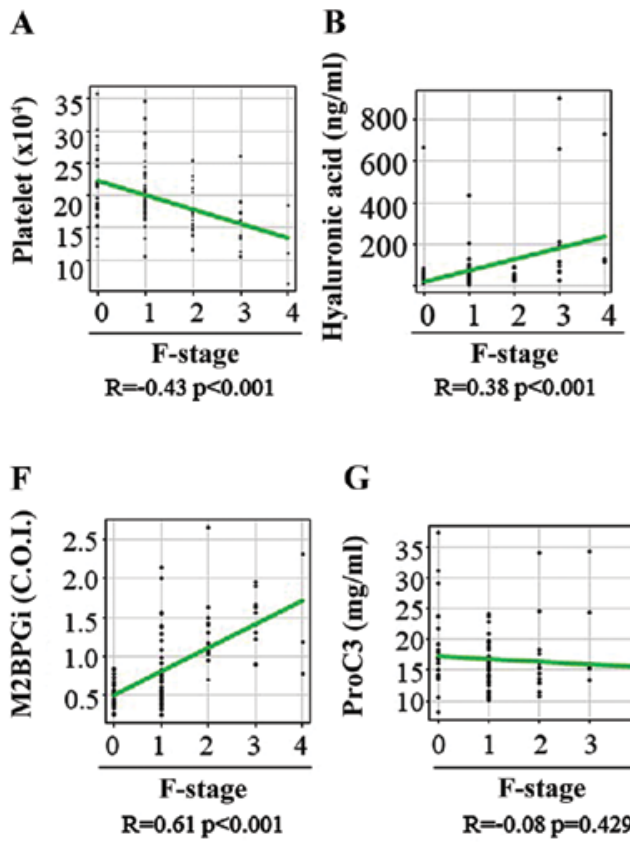

G

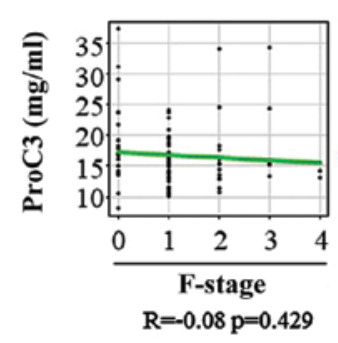

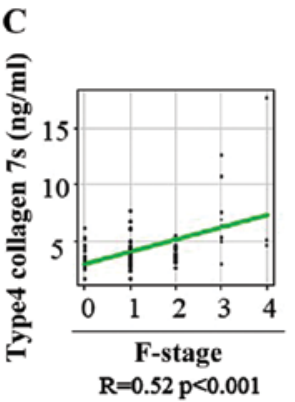

H

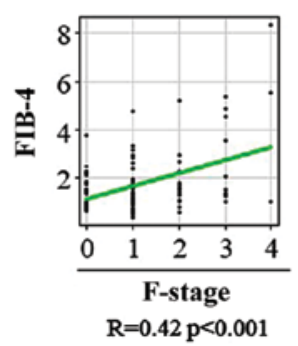

D

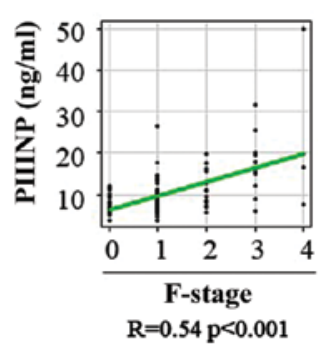

I

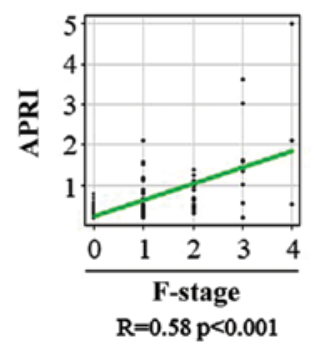

$\mathbf{E}$

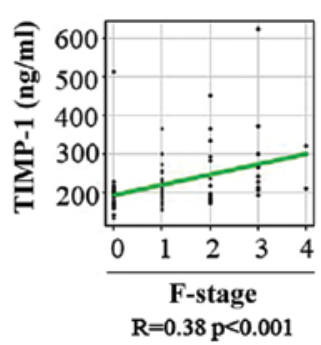

$\mathbf{J}$

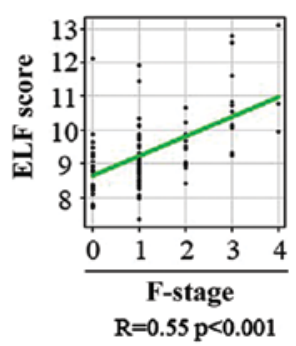

Figure 1. Correlation between 10 liver fibrosis biomarkers and fibrosis stage in patients with chronic hepatitis B. (A) Platelet count, (B) hyaluronan acid, (C) 7S domain of type 4, (D) PIIINP, (E) TIMP-1, (F) M2BPGi, (G) Pro-C3, (H) FIB-4 index, (I) APRI and (J) ELF score. PIIINP, procollagen type III N-terminal peptide; TIMP-1, tissue inhibitor of metalloproteinases 1; M2BPGi, Mac-2 binding protein glycosylation isomer; Pro-C3, N-terminal type III collagen propeptide; FIB-4 index, fibrosis index based on four factors; APRI, aspartate aminotransferase-to-platelet ratio index; ELF, enhanced liver fibrosis; F, fibrosis.

$\mathrm{P}<0.05, \mathrm{P}<0.01, \mathrm{P}<0.001$ and $\mathrm{P}<0.001$, respectively) and no significant difference was found between M2BPGi level and
APRI and ELF score $(\mathrm{P}=0.093$ and $\mathrm{P}=0.072$, respectively) in patients with CHB (Table SI). 
Table III. Diagnostic accuracy of serum fibrosis markers for significant fibrosis in patients with chronic hepatitis B.

\begin{tabular}{|c|c|c|c|c|c|c|c|c|}
\hline Biomarker & AUC & $95 \% \mathrm{CI}$ & Cut-off & $\begin{array}{c}\text { Sensitivity } \\
(95 \% \mathrm{CI})\end{array}$ & $\begin{array}{c}\text { Specificity } \\
(95 \% \text { CI })\end{array}$ & $\begin{array}{c}\text { PPV } \\
(95 \% \mathrm{CI})\end{array}$ & $\begin{array}{c}\text { NPV } \\
(95 \% \mathrm{CI})\end{array}$ & $\begin{array}{l}\text { Accuracy } \\
(95 \% \text { CI })\end{array}$ \\
\hline $\begin{array}{l}\text { Platelet } \\
\text { count }\end{array}$ & 0.757 & $0.641-0.872$ & 17.5 & $\begin{array}{c}70.4 \\
(49.8-86.2)\end{array}$ & $\begin{array}{c}76.8 \\
(65.1-86.1)\end{array}$ & $\begin{array}{c}54.3 \\
(36.6-71.2)\end{array}$ & $\begin{array}{c}86.9 \\
(75.8-94.2)\end{array}$ & $\begin{array}{c}75.0 \\
(65.1-83.3)\end{array}$ \\
\hline $\begin{array}{l}\text { Hyalorinic } \\
\text { acid }\end{array}$ & 0.776 & $0.675-0.877$ & 61.08 & $\begin{array}{c}63.0 \\
(42.4-80.6)\end{array}$ & $\begin{array}{c}84.1 \\
(73.3-91.8)\end{array}$ & $\begin{array}{c}60.7 \\
(40.6-78.5)\end{array}$ & $\begin{array}{c}85.3 \\
(74.6-92.7)\end{array}$ & $\begin{array}{c}78.1 \\
(68.5-85.9)\end{array}$ \\
\hline $\begin{array}{l}\text { Type } 4 \\
\text { collagen } 7 \mathrm{~S}\end{array}$ & 0.739 & $0.621-0.856$ & 4.6 & $\begin{array}{c}59.3 \\
(38.8-77.6)\end{array}$ & $\begin{array}{c}83.6 \\
(72.5-91.5)\end{array}$ & $\begin{array}{c}59.3 \\
(38.8-77.6)\end{array}$ & $\begin{array}{c}83.6 \\
(72.5-91.5)\end{array}$ & $\begin{array}{c}76.6 \\
(66.7-84.7)\end{array}$ \\
\hline PIIINP & 0.778 & $0.668-0.888$ & 15.0 & $\begin{array}{c}48.1 \\
(28.7-68.1)\end{array}$ & $\begin{array}{c}97.1 \\
(89.9-99.6)\end{array}$ & $\begin{array}{c}86.7 \\
(59.5-98.3)\end{array}$ & $\begin{array}{c}82.7 \\
(72.7-90.2)\end{array}$ & $\begin{array}{c}83.3 \\
(74.4-90.2)\end{array}$ \\
\hline TIMP-1 & 0.713 & $0.591-0.833$ & 264.3 & $\begin{array}{c}44.4 \\
(25.5-64.7)\end{array}$ & $\begin{array}{c}94.2 \\
(85.8-98.4)\end{array}$ & $\begin{array}{c}75.0 \\
(47.6-92.7)\end{array}$ & $\begin{array}{c}81.2 \\
(71.0-89.1)\end{array}$ & $\begin{array}{c}80.2 \\
(70.8-87.6)\end{array}$ \\
\hline M2BPGi & 0.902 & $0.841-0.962$ & 0.890 & $\begin{array}{c}92.6 \\
(75.7-99.1)\end{array}$ & $\begin{array}{c}82.4 \\
(71.2-90.5)\end{array}$ & $\begin{array}{c}67.6 \\
(50.2-82.0)\end{array}$ & $\begin{array}{c}96.6 \\
(88.1-99.6)\end{array}$ & $\begin{array}{c}85.3 \\
(76.5-91.7)\end{array}$ \\
\hline Pro-C3 & 0.452 & $0.325-0.579$ & 10.781 & $\begin{array}{c}100 \\
(81.7-100)\end{array}$ & $\begin{array}{c}11.6 \\
(5.1-21.6)\end{array}$ & $\begin{array}{c}30.7 \\
(21.3-41.4)\end{array}$ & $\begin{array}{c}100 \\
(51.8-100)\end{array}$ & $\begin{array}{c}36.5 \\
(26.9-46.9)\end{array}$ \\
\hline FIB-4 & 0.676 & $0.555-0.798$ & 1.264 & $\begin{array}{c}77.8 \\
(57.7-91.4)\end{array}$ & $\begin{array}{c}50.7 \\
(38.4-63.0)\end{array}$ & $\begin{array}{c}38.2 \\
(25.4-52.3)\end{array}$ & $\begin{array}{c}85.4 \\
(70.8-94.4)\end{array}$ & $\begin{array}{c}58.3 \\
(47.8-68.3)\end{array}$ \\
\hline APRI & 0.812 & $0.709-0.914$ & 0.534 & $\begin{array}{c}81.5 \\
(61.9-93.7)\end{array}$ & $\begin{array}{c}75.4 \\
(63.5-84.9)\end{array}$ & $\begin{array}{c}56.4 \\
(39.6-72.2)\end{array}$ & $\begin{array}{c}91.2 \\
(80.7-97.1)\end{array}$ & $\begin{array}{c}77.1 \\
(67.4-85.0)\end{array}$ \\
\hline ELF score & 0.816 & $0.723-0.909$ & 9.250 & $\begin{array}{c}77.8 \\
(75.7-91.4)\end{array}$ & $\begin{array}{c}73.9 \\
(61.9-83.7)\end{array}$ & $\begin{array}{c}53.8 \\
(37.2-69.9)\end{array}$ & $\begin{array}{c}89.5 \\
(78.5-96.0)\end{array}$ & $\begin{array}{c}75.0 \\
(65.1-83.3)\end{array}$ \\
\hline
\end{tabular}

PIIINP, type 3 procollagen-N-peptide; TIMP-1, tissue inhibitor of metalloproteinase 1; M2BPGi, Mac-2-binding protein glycosylation isomer; Pro-C3, N-terminal type III collagen propeptide; FIB-4, the fibrosis index based on four factors; APRI, the aspartate aminotransferase-to-platelet ratio index; ELF score, enhanced liver fibrosis score.
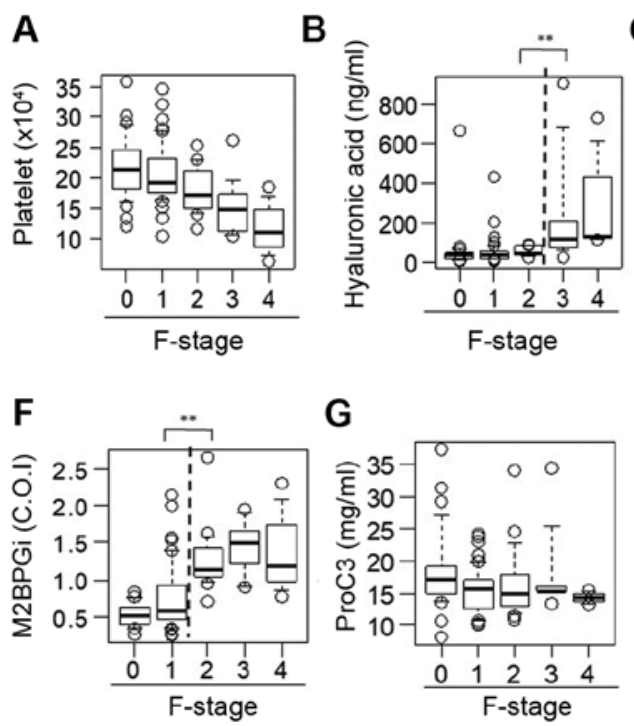
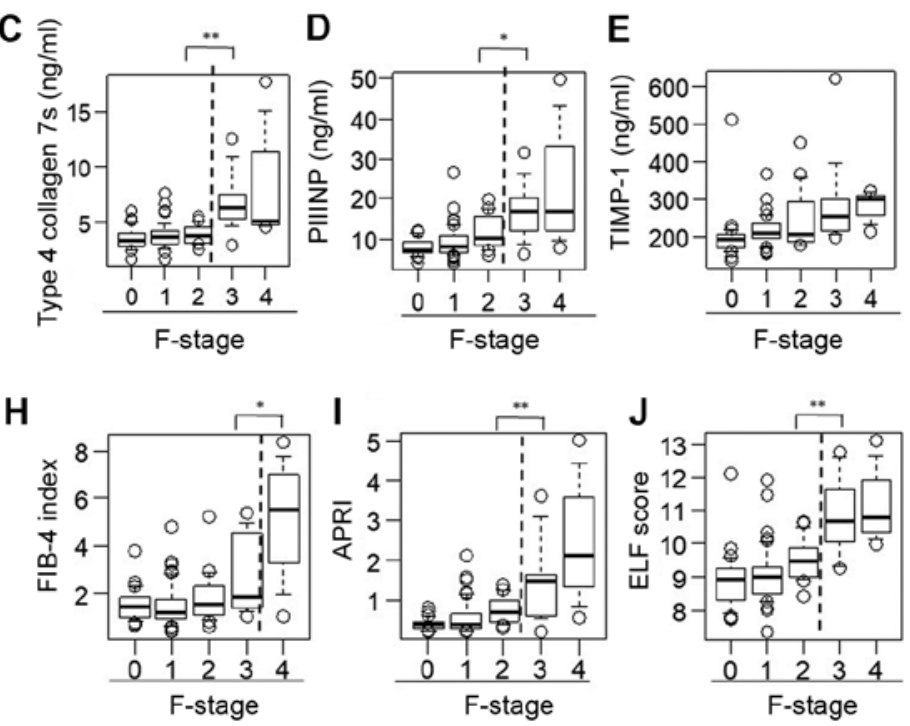

Figure 2. Serum levels of fibrosis biomarkers for staging liver fibrosis in patients with chronic hepatitis B. A box plot of each fibrosis marker according to the fibrosis stage is presented. (A) Platelet count, (B) hyaluronan acid, (C) 7S domain of type 4, (D) PIIINP, (E) TIMP-1, (F) M2BPGi, (G) Pro-C3, (H) FIB-4 index, (I) APRI and ( $\mathrm{J})$ ELF score. Data are presented as mean \pm standard deviation. ${ }^{*} \mathrm{P}<0.05$ and ${ }^{* *} \mathrm{P}<0.01$ as indicated. M2BPGi, Mac-2 binding protein glycosylation isomer; PIIINP, procollagen type III N-terminal peptide; APRI, aspartate aminotransferase-to-platelet ratio index; ELF, enhanced liver fibrosis; FIB-4 index, fibrosis index based on four factors; TIMP-1, tissue inhibitor of metalloproteinases 1; Pro-C3, N-terminal type III collagen propeptide; F, fibrosis.

Association of the M2BPGi level with the fibrosis stage in terms of histological necroinflammatory activity. The ALT level was significantly higher in patients with significant liver necroinflammation $(n=23)$ compared with those without significant necroinflammation $(\mathrm{n}=73$; $\mathrm{P}<0.01$; Table SII). A significant correlation was observed between the M2BPGi 


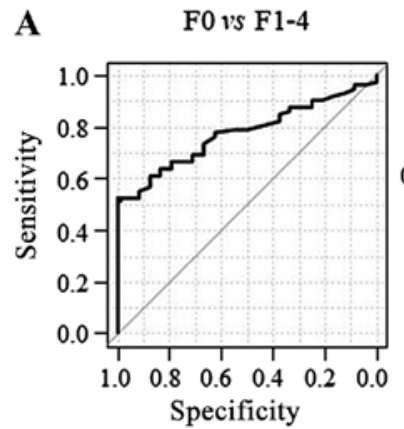

$0.890(1.000,0.521)$ AUC: 0.773

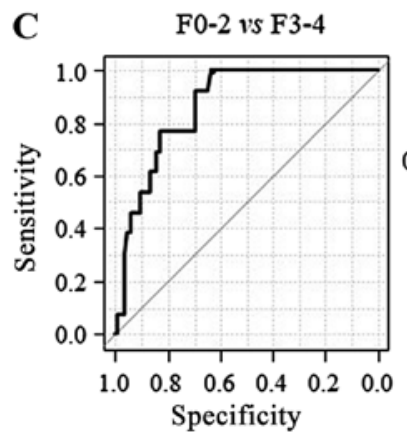

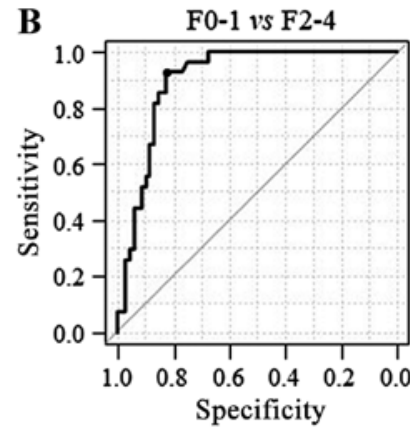

$0.890(0.824,0.926)$

AUC: 0.902

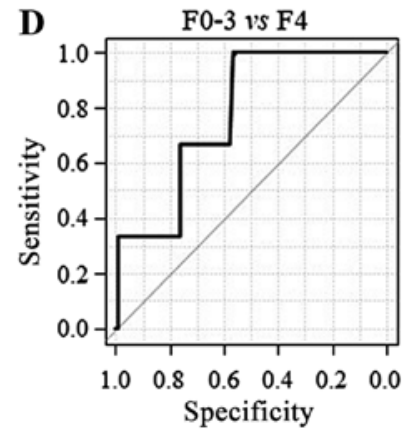

$0.770(0.565,1.000)$

AUC: 0.774

Figure 3. Diagnostic performance of serum M2BPGi for identifying the different stages of liver fibrosis in patients with chronic hepatitis B. Receiver operating characteristic curve analysis and AUC for the diagnostic performance of serum M2BPGi for identifying different stages of liver fibrosis. (A) F0 vs. F1-4, (B) F0-1 vs. F2-4 (C) F0-2 vs. F3-4 and (D) F0-3 vs. F4. M2BPGi, Mac-2 binding protein glycosylation isomer; AUC, area under the curve; F, fibrosis stage.
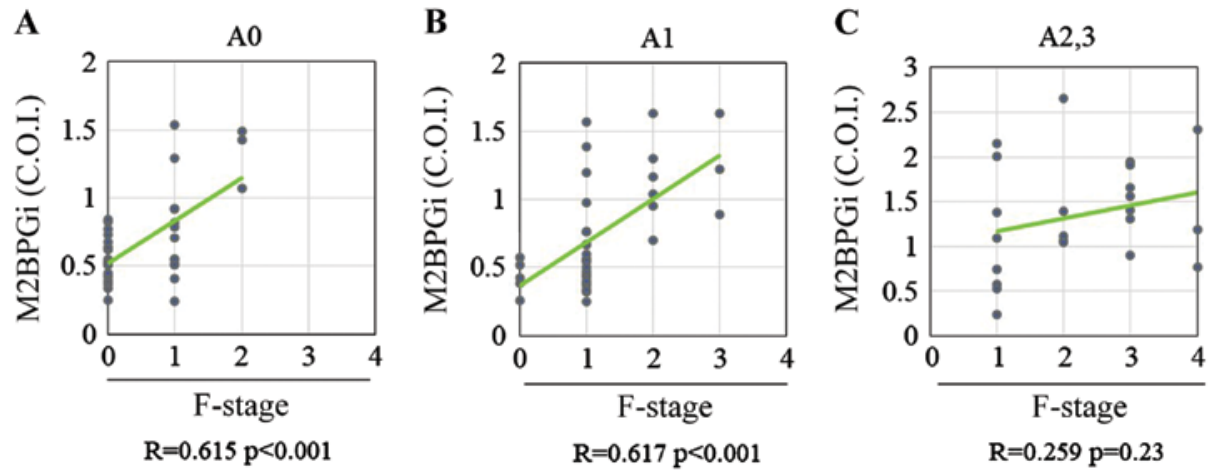

Figure 4. Correlation of the M2BPGi level with the fibrosis stage in terms of liver necroinflammation activity. (A and B) Significant correlation between the M2BPGi level and fibrosis stage is noted in patients with chronic hepatitis B without significant liver necroinflammation, A0-A1. (C) No correlation was observed in patients with CHB with significant liver necroinflammation, A2 or A3. COI, cutoff index; M2BPGi, Mac-2 binding protein glycosylation isomer; F, fibrosis.

level and fibrosis stage in patients with $\mathrm{CHB}$ without significant liver necroinflammation (A0-1; Fig. 4A and B), whereas no significant correlation was observed between these two variables in those with significant liver necroinflammation $(\geq A 2$; Fig. 4C). These findings indicated that significant liver necroinflammation might affect the M2BPGi level in patients with $\mathrm{CHB}$. Among patients with $\mathrm{CHB}$ without significant liver necroinflammation, Spearman's rank correlation coefficients between the fibrosis stage and PLT count, HA level, 7S collagen level, PIIINP level, TIMP-1 level, M2BPGi level, Pro-C3 level, FIB-4 index, APRI and ELF score were $-0.31,0.04,0.21,0.20,0.15,0.59,-0.10,0.19$, 0.28 and 0.26 , respectively (Fig. 5A-J). The PLT count and M2BPGi level were the only variables associated with the liver fibrosis stage in patients with CHB without significant liver necroinflammation (Table IV).
Levels of serum fibrosis biomarkers according to the degree of liver fibrosis in patients with CHB without significant liver necroinflammation. Significant differences were observed between patients with F1 fibrosis and those with F2 fibrosis with regard to the PLT count (F1, 21.0 \pm 5.0 vs. F2, $16.4 \pm 3.2 \times 10^{4} / \mu 1 ; \mathrm{P}<0.01$; Fig. $\left.6 \mathrm{~A}\right)$ and M2BPGi level (F1, $0.68 \pm 0.34$ vs. F2, $1.20 \pm 0.28$ COI; P<0.01; Fig. 6B). There were significant differences between patients with $\mathrm{F} 2$ fibrosis and those with F3 fibrosis with regard to the TIMP-1 level (F2, $193.5 \pm 19.5$ vs. $\mathrm{F} 3,264.4 \pm 77.7 \mathrm{ng} / \mathrm{ml} ; \mathrm{P}<0.01$; Fig. 6C), ELF score (F2, 9.21 \pm 0.52 vs. F3, 10.20 \pm 0.69 ; $\mathrm{P}<0.05$; Fig. 6D) and HA level (F2, 47.4 \pm 23.1 vs. F3, $123.9 \pm 51.9 \mathrm{ng} / \mathrm{ml}$; P<0.01; Fig. 6E). However, no differences were observed among the fibrosis groups with regard to the 7S collagen level, PIIINP level, Pro-C3 level, FIB-4 index and APRI (Fig. 6F-J). 
A

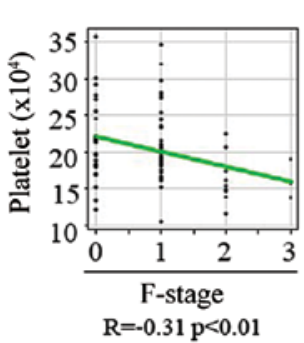

F

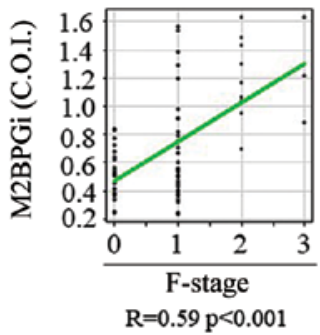

B

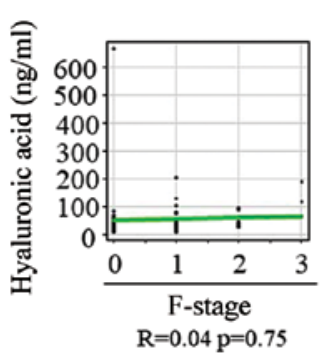

G

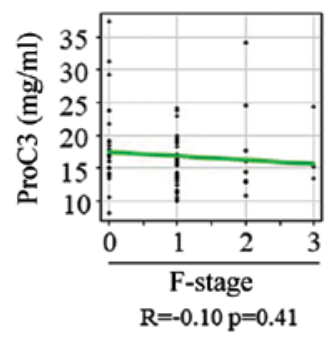

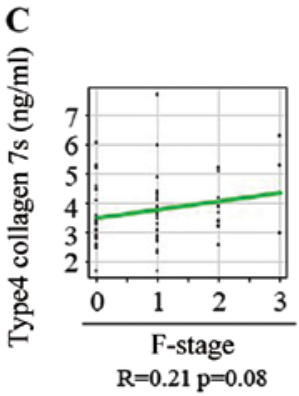

H

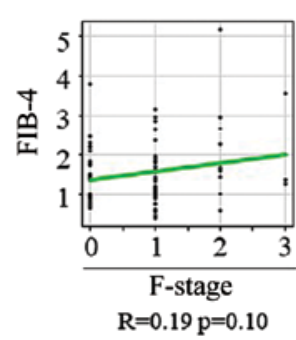

D

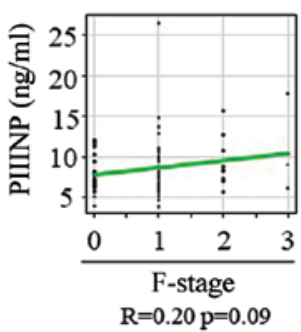

I

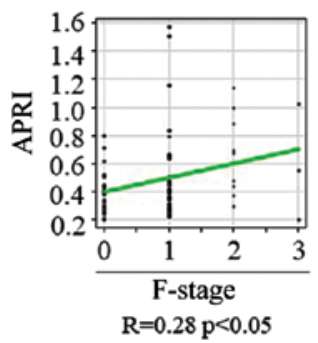

E

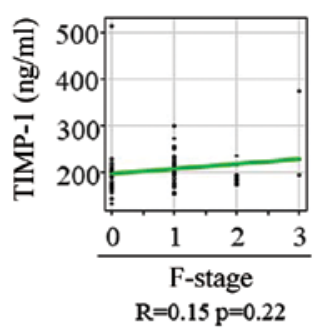

J

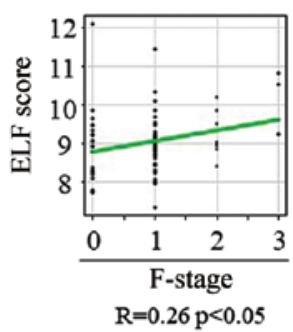

Figure 5. Correlation between 10 liver fibrosis biomarkers and fibrosis stage in patients with chronic hepatitis B without significant liver necroinflammation. (A) Platelet count, (B) hyaluronan acid, (C) 7S domain of type 4, (D) PIIINP; (E) TIMP-1, (F) M2BPGi, (G) Pro-C3, (H) FIB-4 index, (I) APRI and (J) ELF score. PIIINP, procollagen type III N-terminal peptide; TIMP-1, tissue inhibitor of metalloproteinases 1; M2BPGi, Mac-2 binding protein glycosylation isomer; Pro-C3, N-terminal type III collagen propeptide; FIB-4 index, fibrosis index based on four factors; APRI, aspartate aminotransferase-to-platelet ratio index; ELF, enhanced liver fibrosis; F, fibrosis.

Diagnostic performances of serum fibrosis biomarkers for identifying significant liver fibrosis in patients with $C H B$ without significant liver necroinflammation. The optimal cut-off value and diagnostic performance of each serum fibrosis biomarker for identifying significant liver fibrosis in patients with $\mathrm{CHB}$ without significant liver necroinflammation are presented in Table V. The AUCs of the PLT count, HA level, 7S collagen level, PIIINP level, TIMP-1 level, M2BPGi level, Pro-C3 level, FIB-4 index, APRI and ELF score for the correct diagnosis of significant liver fibrosis were 0.807, 0.678, 0.603, $0.632,0.501,0.921,0.458,0.678,0.708$ and 0.688 , respectively. These findings indicated that the serum M2BPGi level more accurately identified significant liver fibrosis when compared with other non-invasive fibrosis biomarkers in patients with CHB without significant liver necroinflammation.

Diagnostic performance of serum M2BPGi for identifying the different stages of liver fibrosis in patients with $C H B$ without significant liver necroinflammation. The AUCs of the M2BPGi level for identifying F1-3, F2-3 and F3 were 0.704, 0921 and 0.882 , respectively (Fig. 7), indicating that M2BPGi exhibited a higher diagnostic accuracy for significant fibrosis than mild or severe fibrosis. These results suggested that serum M2BPGi had the best performance for identifying significant liver fibrosis in patients with $\mathrm{CHB}$ who had significant liver fibrosis but did not have significant liver necroinflammation. In addition, significant differences were identified in the AUCs between M2BPGi level and HA level, 7S collagen level, PIIINP level, TIMP-1 level, Pro-C3 level, FIB-4 index, APRI and ELF score $(\mathrm{P}<0.01, \mathrm{P}<0.05, \mathrm{P}<0.01, \mathrm{P}<0.001, \mathrm{P}<0.001$, $\mathrm{P}<0.05, \mathrm{P}<0.05$ and $\mathrm{P}<0.05$, respectively) but not between M2BPGi level and PLT count in patients with CHB without significant liver necroinflammation (Table SI).

\section{Discussion}

Liver fibrosis staging plays an important role in the selection of patients with CHB for antiviral therapy and liver biopsy is a reference tool for the decision to start therapy. However, biopsy has limitations, such as high cost, invasiveness, bleeding complications and sampling variability. The serum biomarkers of liver fibrosis are considered to have limited diagnostic utility (22). There is no established biomarker for patients with $\mathrm{CHB}$ who require antiviral therapy. To the best of the authors' knowledge, the present study is the first to report that the serum M2BPGi level is a useful marker for identifying liver histological findings in patients with $\mathrm{CHB}$ without significant necroinflammation and in need of antiviral therapy.

M2BPGi is a glycosylated secretory protein synthesized by activated hepatic stellate cells (Ac-HSCs) and is emerging as a serum marker for liver fibrosis (26). M2BPGi serves as a juxtacrine messenger between Ac-HSCs and Kupffer cells during progression of liver fibrosis (27). Bekki et al (28) demonstrated that M2BPGi is exclusively produced in Ac-HSCs and plays an important role in the progression of liver fibrosis. M2BPGi has been recently developed as a novel serum biomarker that is strongly correlated with liver fibrosis in patients with CHC (29). Several studies have identified that M2BPGi can serve as a serum fibrosis marker in patients with CHB (30-33), although M2BPGi levels vary for the same fibrosis stage between patients with $\mathrm{CHB}$ and $\mathrm{CHC}$ (31). This may be partly explained by the fact that the generative nodule size and fibrous septum thickness (composed of collagen fibrils) substantially differ between patients with CHB and CHC (34). These findings support the potential role of M2BPGi as a surrogate biomarker that reflects hepatic stellate cell function (28). 
Table IV. Baseline characteristics of patients with chronic hepatitis B without significant liver inflammation stratified according to liver fibrosis stages.

\begin{tabular}{|c|c|c|c|c|c|}
\hline Variable (reference range) & $\mathrm{F} 0(\mathrm{n}=25)$ & $\mathrm{F} 1(\mathrm{n}=36)$ & $\mathrm{F} 2(\mathrm{n}=9)$ & $\mathrm{F} 3(\mathrm{n}=3)$ & Overall P-value \\
\hline Male/female & $11 / 14$ & $19 / 17$ & $3 / 6$ & $2 / 1$ & NS \\
\hline Age (years) & $53.7 \pm 2.4$ & $50.0 \pm 2.3$ & $51.2 \pm 4.6$ & $55.9 \pm 8.0$ & NS \\
\hline Platelet count $\left(10^{4} / \mu 1\right)(10.4-37.9)$ & $21.6 \pm 1.1$ & $21.0 \pm 0.8^{\mathrm{b}}$ & $16.4 \pm 1.1$ & $16.4 \pm 1.2$ & 0.03 \\
\hline AST (IU/l) (10-40) & $26.1 \pm 2.6$ & $31.3 \pm 3.4$ & $31.9 \pm 3.4$ & $27.2 \pm 7.6$ & NS \\
\hline $\operatorname{ALT}(\mathrm{IU} / 1)(5-45)$ & $22.0 \pm 2.4$ & $38.3 \pm 6.0$ & $30.0 \pm 5.0$ & $22.7 \pm 6.2$ & NS \\
\hline Serum albumin $(\mathrm{g} / \mathrm{dl})(3.7-5.5)$ & $4.2 \pm 0.1$ & $4.2 \pm 0.1$ & $4.0 \pm 0.1$ & $4.4 \pm 0.2$ & NS \\
\hline Total Bilirubin (mg/dl) (0.3-1.2) & $0.8 \pm 0.0$ & $0.8 \pm 0.1$ & $0.9 \pm 0.1$ & $1.0 \pm 0.2$ & NS \\
\hline HBV DNA (Log copies/ml) & $3.5 \pm 0.4$ & $4.5 \pm 0.4$ & $5.5 \pm 0.6$ & $3.4 \pm 1.4$ & NS \\
\hline HBsAg (IU/ml) $(<0.05)$ & $12,128 \pm 5,088$ & $41,852 \pm 2,863$ & $10,580 \pm 8,840$ & $1,602 \pm 520$ & NS \\
\hline Hyaluronic acid (ng/ml) $(<50.0)$ & $61.7 \pm 25.8$ & $45.0 \pm 6.1$ & $47.4 \pm 7.7$ & $123.9 \pm 30.0^{\mathrm{c}}$ & NS \\
\hline Type 4 collagen $7 \mathrm{~S}(\mathrm{ng} / \mathrm{ml})(<6.0)$ & $3.6 \pm 0.2$ & $3.8 \pm 0.1$ & $3.8 \pm 0.3$ & $4.9 \pm 0.8^{c}$ & NS \\
\hline PIIINP (ng/ml) (3.6-9.52) & $7.9 \pm 0.5$ & $8.7 \pm 7.3$ & $9.4 \pm 1.0$ & $11.0 \pm 2.9$ & NS \\
\hline TIMP-1 (ng/ml) & $198.6 \pm 13.8$ & $211.1 \pm 5.7$ & $193.5 \pm 6.5^{c}$ & $264.4 \pm 45.0$ & NS \\
\hline M2BPGi (COI) $(<1.00)$ & $0.53 \pm 0.32$ & $0.68 \pm 0.6^{\mathrm{b}}$ & $1.20 \pm 0.1$ & $1.25 \pm 0.17$ & $<0.01$ \\
\hline ProC3 (ng/ml) & $18.5 \pm 1.3$ & $15.8 \pm 0.6$ & $17.4 \pm 2.3$ & $17.7 \pm 2.8$ & NS \\
\hline FIB-4 & $1.52 \pm 0.14$ & $1.38 \pm 0.12$ & $2.15 \pm 0.43$ & $2.07 \pm 0.61$ & NS \\
\hline APRI & $0.43 \pm 0.05$ & $0.50 \pm 0.05$ & $0.66 \pm 0.09$ & $0.59 \pm 0.20$ & NS \\
\hline ELF score & $8.91 \pm 0.18$ & $8.99 \pm 0.12^{\mathrm{c}}$ & $9.21 \pm 0.17$ & $10.20 \pm 0.40^{\mathrm{d}}$ & NS \\
\hline
\end{tabular}

${ }^{b} \mathrm{P}<0.01$ vs. $\mathrm{F} 2$; ${ }^{\mathrm{c}} \mathrm{P}<0.01$ vs. F3; ${ }^{\mathrm{d}} \mathrm{P}<0.01$ vs. F4; AST, aspartate aminotransferase; ALT, alanine aminotransferase; PIIINP, type 3 procollagen-N-peptide; TIMP-1, tissue inhibitor of metalloproteinase 1; M2BPGi, Mac-2-binding protein glycosylation isomer; Pro-C3, N-terminal type III collagen propeptide; FIB-4, fibrosis index based on four factors; APRI, aspartate aminotransferase-to-platelet ratio index; ELF score, enhanced liver fibrosis score; F, fibrosis stage.

A
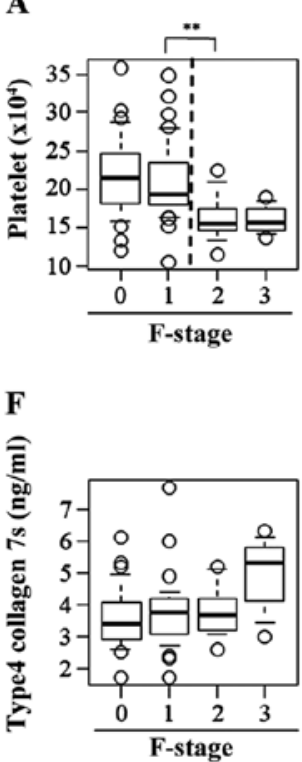

B

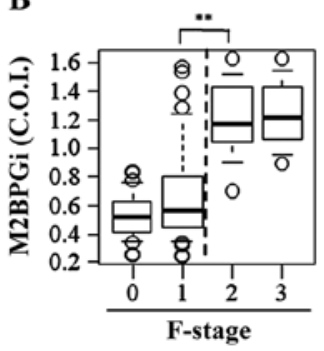

G

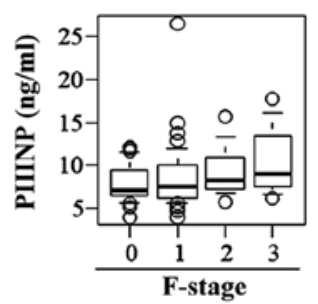

C

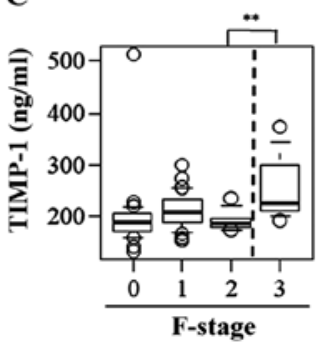

H

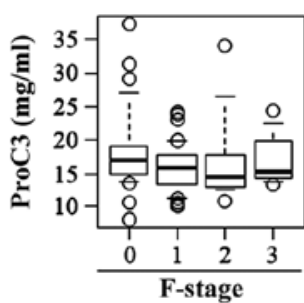

D

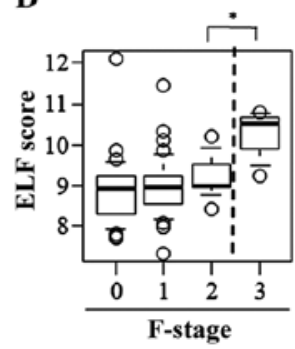

I

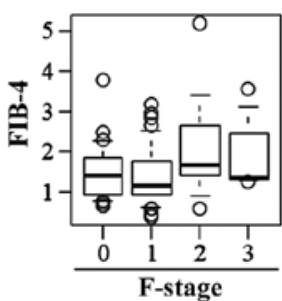

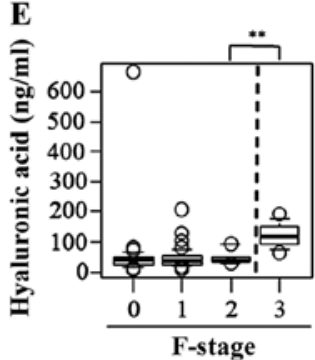

$\mathbf{J}$

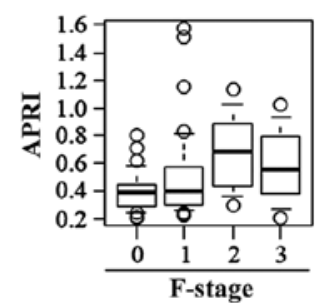

Figure 6. Serum levels of fibrosis biomarkers for staging liver fibrosis in patients with chronic hepatitis B without significant liver necroinflammation. A box plot of each fibrosis marker according to the fibrosis stage is presented. (A) Platelet count, (B) M2BPGi, (C) TIMP-1, (D) ELF score, (E) hyaluronan acid, (F) 7S domain of type 4 collagen, (G) PIIINP, (H) Pro-C3, (I) FIB-4 index and (J) APRI. Data are presented as mean \pm standard deviation. ${ }^{*} \mathrm{P}<0.05$ and ${ }^{* *} \mathrm{P}<0.01$ as indicated. M2BPGi, Mac-2 binding protein glycosylation isomer; TIMP-1, tissue inhibitor of metalloproteinases 1; ELF, enhanced liver fibrosis; PIIINP, procollagen type III N-terminal peptide; Pro-C3, N-terminal type III collagen propeptide; FIB-4 index, fibrosis index based on four factors; APRI, aspartate aminotransferase-to-platelet ratio index; F, fibrosis.

M2BPGi levels have been found to rapidly decrease with reduced hepatic inflammation during direct-acting antiviral therapy for HCV infection in patients with $\mathrm{CHC}(27,35)$. In agreement with the current findings, serum M2BPGi 
Table V. Diagnostic accuracy of serum fibrosis markers for significant fibrosis in patients with chronic hepatitis B without significant liver inflammation.

\begin{tabular}{|c|c|c|c|c|c|c|c|c|}
\hline Biomarker & AUC & $95 \%$ CI & Cut-off & $\begin{array}{l}\text { Sensitivity } \\
(95 \% \text { CI })\end{array}$ & $\begin{array}{l}\text { Specificity } \\
(95 \% \text { CI })\end{array}$ & $\begin{array}{c}\text { PPV } \\
(95 \% \text { CI })\end{array}$ & $\begin{array}{c}\text { NPV } \\
(95 \% \mathrm{CI})\end{array}$ & $\begin{array}{l}\text { Accuracy } \\
(95 \% \text { CI) }\end{array}$ \\
\hline Platelet & 0.807 & $0.670-0.947$ & 16.2 & $\begin{array}{c}66.7 \\
(34.9-90.1)\end{array}$ & $\begin{array}{c}88.5 \\
(77.8-95.3)\end{array}$ & $\begin{array}{c}53.3 \\
(16.6-78.7\end{array}$ & $\begin{array}{c}93.1 \\
(83.3-98.1)\end{array}$ & $\begin{array}{c}84.9 \\
(74.6-92.2)\end{array}$ \\
\hline $\begin{array}{l}\text { Hyaluronic } \\
\text { acid }\end{array}$ & 0.678 & $0.522-0.833$ & 26.6 & $\begin{array}{c}100 \\
(64.0-100)\end{array}$ & $\begin{array}{c}37.7 \\
(25.6-51.0)\end{array}$ & $\begin{array}{c}24.0 \\
(13.1-38.2)\end{array}$ & $\begin{array}{c}100 \\
(78.9-100)\end{array}$ & $\begin{array}{c}47.9 \\
(36.1-60.0)\end{array}$ \\
\hline $\begin{array}{l}\text { Type } 4 \\
\text { collagen } 7 \mathrm{~S}\end{array}$ & 0.603 & $0.416-0.791$ & 5.1 & $\begin{array}{c}33.3 \\
(9.9-65.1)\end{array}$ & $\begin{array}{c}91.8 \\
(81.9-97.3)\end{array}$ & $\begin{array}{c}44.4 \\
(13.7-78.8)\end{array}$ & $\begin{array}{c}87.5 \\
(76.8-94.4)\end{array}$ & $\begin{array}{c}82.2 \\
(71.5-90.2)\end{array}$ \\
\hline PIIINP & 0.632 & $0.454-0.810$ & 8.27 & $\begin{array}{c}66.7 \\
(34.9-90.1)\end{array}$ & $\begin{array}{c}65.6 \\
(52.3-77.3)\end{array}$ & $\begin{array}{c}27.6 \\
(12.7-47.2)\end{array}$ & $\begin{array}{c}90.9 \\
(78.3-97.5)\end{array}$ & $\begin{array}{c}65.8 \\
(53.7-76.5)\end{array}$ \\
\hline TIMP-1 & 0.501 & $0.329-0.674$ & 174.7 & $\begin{array}{c}100 \\
(64.0-100)\end{array}$ & $\begin{array}{c}24.6 \\
(14.5-37.3)\end{array}$ & $\begin{array}{c}20.7 \\
(11.2-33.4)\end{array}$ & $\begin{array}{c}100 \\
(69.8-100)\end{array}$ & $\begin{array}{c}37.0 \\
(26.0-49.1)\end{array}$ \\
\hline M2BPGi & 0.921 & $0.856-0.986$ & 0.890 & $\begin{array}{c}91.7 \\
(61.5-99.8)\end{array}$ & $\begin{array}{c}86.7 \\
(75.4-94.1)\end{array}$ & $\begin{array}{c}57.9 \\
(33.5-79.7)\end{array}$ & $\begin{array}{c}98.1 \\
(89.9-100)\end{array}$ & $\begin{array}{c}87.5 \\
(77.6-94.1)\end{array}$ \\
\hline Pro-C3 & 0.458 & $0251-0.664$ & 24.35 & $\begin{array}{c}25.0 \\
(5.5-57.2)\end{array}$ & $\begin{array}{c}95.1 \\
(86.3-99.0)\end{array}$ & $\begin{array}{c}50.0 \\
(11.8-88.2)\end{array}$ & $\begin{array}{c}86.6 \\
(76.0-93.7)\end{array}$ & $\begin{array}{c}83.6 \\
(73.0-91.2)\end{array}$ \\
\hline FIB-4 & 0.678 & $0.500-0.855$ & 1.264 & $\begin{array}{c}83.3 \\
(51.6-97.9)\end{array}$ & $\begin{array}{c}50.8 \\
(37.7-63.9)\end{array}$ & $\begin{array}{c}25.0 \\
(12.7-41.2)\end{array}$ & $\begin{array}{c}93.9 \\
(79.8-93.3)\end{array}$ & $\begin{array}{c}56.2 \\
(44.1-67.8)\end{array}$ \\
\hline APRI & 0.708 & $0.524-0.892$ & 0.438 & $\begin{array}{c}75.0 \\
(42.8-94.5)\end{array}$ & $\begin{array}{c}65.6 \\
(52.3-77.3)\end{array}$ & $\begin{array}{c}30.0 \\
(14.7-49.4)\end{array}$ & $\begin{array}{c}93.0 \\
(80.9-98.5)\end{array}$ & $\begin{array}{c}67.1 \\
(55.1-77.7)\end{array}$ \\
\hline ELF score & 0.688 & $0.530-0.846$ & 8.87 & $\begin{array}{c}91.7 \\
(61.5-99.8)\end{array}$ & $\begin{array}{c}44.3 \\
(31.5-57.6)\end{array}$ & $\begin{array}{c}24.4 \\
(12.9-39.5)\end{array}$ & $\begin{array}{c}96.4 \\
(81.7-99.9)\end{array}$ & $\begin{array}{c}52.1 \\
(40.0-63.9)\end{array}$ \\
\hline
\end{tabular}

PIIINP, type 3 procollagen-N-peptide; TIMP-1, tissue inhibitor of metalloproteinase 1; M2BPGi, Mac-2-binding protein glycosylation isomer; Pro-C3, N-terminal type III collagen propeptide; FIB-4, fibrosis index based on four factors; APRI, aspartate aminotransferase-to-platelet ratio index; ELF score, enhanced liver fibrosis score.
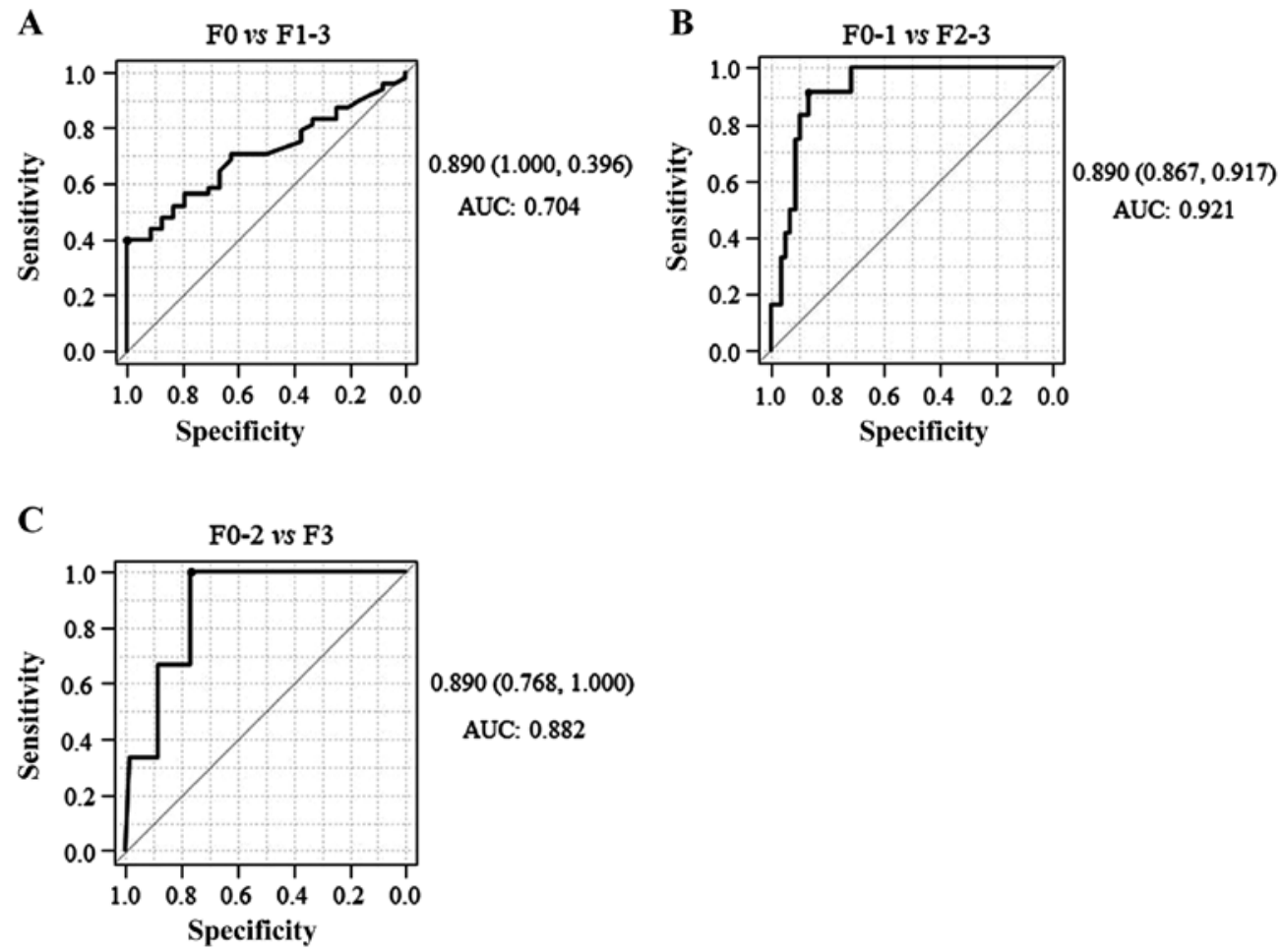

Figure 7. Diagnostic performance of serum M2BPGi for identifying the different stages of liver fibrosis in patients with chronic hepatitis B without significant liver necroinflammation. Receiver operating characteristic curve analysis and AUC for the diagnostic performance of serum M2BPGi for identifying the different stages of liver fibrosis. (A) F0 vs. F1-3, (B) F0-1 vs. F2-3 and (C) F0-2 vs. F3. M2BPGi, Mac-2 binding protein glycosylation isomer; AUC, area under the curve; F, fibrosis. 
levels were significantly higher in patients with $\mathrm{CHB}$ with significant liver necroinflammation than in those without significant liver necroinflammation (36). All serum fibrosis biomarkers, except the Pro-C3 level, were correlated with the fibrosis stage in all patients with CHB, whereas the PLT count and M2BPGi level were exclusively associated with the fibrosis stage in patients with $\mathrm{CHB}$ without significant liver necroinflammation. In addition, in a previous study, the M2BPGi level was found to be correlated with the serum $\mathrm{C}-\mathrm{X}-\mathrm{C}$ motif chemokine 10 level, which is closely related to the migration of inflammatory cells to the local focus in the liver (30). These results further supported the hypothesis that fibrosis markers are substantially affected by liver inflammation. However, Miyaki et al (32) and Liu et al (33) reported that $\mathrm{M} 2 \mathrm{BPGi}$ levels reflect fibrosis progression and are not affected by inflammation or ALT fluctuations in treatment-naïve patients with $\mathrm{CHB}$. The reasons for the different results between the studies remain unclear. However, one possible explanation is the difference in the percentage of patients with cirrhosis between the studies, which warrants further investigation.

The present study found that the M2BPGi level had the highest diagnostic performance for identifying significant liver fibrosis in patients with CHB without significant necroinflammation. Recently, the ELF score and M2BPGi level demonstrated comparable diagnostic performances for identifying significant liver fibrosis in patients with CHB (32). A recent report by Jekarl et al (20) demonstrated that the PLT count, ELF score and M2BPGi level accurately identified significant liver fibrosis in treatment-naïve patients with CHB. Serum M2BPGi has been shown to have a good performance for diagnosing severe fibrosis in patients with CHB treated with nucleoside analogs (33). Mak et al (37) demonstrated that M2BPGi was significantly correlated with severe fibrosis and cirrhosis in patients with $\mathrm{CHB}$ treated with nucleoside analogs. The difference in the ability of serum M2BPGi to identify the liver fibrosis stage between the studies might be attributed to the patient distribution according to the fibrosis stage at inclusion and the administration of nucleoside analogs that might reduce hepatic fibrosis, in addition to the influence of hepatic inflammation. The current results confirm that M2BPGi is a novel non-invasive diagnostic biomarker that can identify treatment-naïve patients with CHB in need of treatment.

The present study has several limitations. First, this was a retrospective study. Second, liver biopsy has the drawback of being prone to sampling errors in fibrosis staging and inflammation grading, potentially leading to bias. Third, the sample size (especially the number of patients with cirrhosis) was small for analysis. It is difficult to obtain a representative liver biopsy specimen from patients with cirrhosis whose platelet counts are lower than $5 \times 10^{4} / \mu 1$. Thus, further research with a large number of patients is required to validate the use of serum M2BPGi level in the detection of significant fibrosis in patients with $\mathrm{CHB}$.

In conclusion, serum M2BPGi level is a useful marker for identifying liver histological findings in patients with $\mathrm{CHB}$ without significant necroinflammation in need of antiviral therapy, although M2BPGi level not only identifies the status of liver fibrosis but also reflects liver necroinflammation (27).

\section{Acknowledgements}

Not applicable.

\section{Funding}

Not applicable.

\section{Availability of data and materials}

The datasets used and/or analyzed during the current study are available from the corresponding author on reasonable request.

\section{Authors' contributions}

YT, KKa, HT, KN, SSat, SSai, YS, KKi, NS, HK, KM, RN, TA and AM performed data analysis. AM supervised all statistical analyses performed in this study. HY and TN made substantial contributions to the conception and design of the study and analysis and interpretation of the data. All authors read and approved the final manuscript.

\section{Ethics approval and consent to participate}

Written informed consent for the use of resected tissue was obtained from all patients and the study protocol was approved by the Ethics Committee of Nara Medical University (approval no. 1077).

\section{Patient consent for publication}

Not applicable.

\section{Competing interests}

The authors declare that they have no competing interests.

\section{References}

1. Okura Y, Namisaki T, Moriya K, Kitade M, Takeda K, Kaji K, Noguchi R, Nishimura N, Seki K, Kawaratani H, et al: Combined treatment with dipeptidyl peptidase-4 inhibitor (sitagliptin) and angiotensin-II type 1 receptor blocker (losartan) suppresses progression in a non-diabetic rat model of steatohepatitis. Hepatol Res 47: 1317-1328, 2017.

2. Saleh HA and Abu-Rashed AH: Liver biopsy remains the gold standard for evaluation of chronic hepatitis and fibrosis. J Gastrointestin Liver Dis 16: 425-426, 2007.

3. Fujinaga Y, Namisaki T, Moriya K, Kitade M, Kawaratani H, Shimozato N, Kaji K, Takaya H, Sawada Y, Seki K, et al: Identification of clinical risk factors for histological progression of primary biliary cholangitis. Hepatol Res 49: 1015-1025, 2019.

4. Venkatesh SK, Yin M, Takahashi N, Glockner JF, Talwalkar JA and Ehman RL: Non-invasive detection of liver fibrosis: MR imaging features vs. MR elastography. Abdom Imaging 40: 766-775, 2015.

5. Mattos AZ and Mattos AA: Transient elastography vs. aspartate aminotransferase to platelet ratio index in hepatitis $\mathrm{C}$ : A meta-analysis. Ann Hepatol 16: 349-357, 2017.

6. Yasui Y, Abe T,Kurosaki M, Matsunaga K, Higuchi M, Tamaki N, Watakabe K, Okada M, Wang W, Shimizu T, et al: Non-invasive liver fibrosis assessment correlates with collagen and elastic fiber quantity in patients with hepatitis $\mathrm{C}$ virus infection. Hepatol Res 49: 33-41, 2019. 
7. Regev A, Berho M, Jeffers LJ, Milikowski C, Molina EG, Pyrsopoulos NT, Feng ZZ, Reddy KR and Schiff ER: Sampling error and intraobserver variation in liver biopsy in patients with chronic HCV infection. Am J Gastroenterol 97: 2614-2618, 2002.

8. Baranova A, Lal P, Birerdinc A and Younossi ZM: Non-invasive markers for hepatic fibrosis. BMC Gastroenterol 11: 91, 2011.

9. Parikh P, Ryan JD and Tsochatzis EA: Fibrosis assessment in patients with chronic hepatitis B virus (HBV) infection. Ann Transl Med 5: 40, 2017.

10. Terrault NA, Lok ASF, McMahon BJ, Chang KM, Hwang JP, Jonas MM, Brown RS Jr, Bzowej NH and Wong JB: Update on prevention, diagnosis, and treatment of chronic hepatitis B: AASLD 2018 hepatitis B guidance. Hepatology 67: 1560-1599, 2018.

11. Drafting Committee for Hepatitis Management Guidelines and the Japan Society of Hepatology: JSH Guidelines for the management of hepatitis B virus infection. Hepatol Res 44 (Suppl 1): S1-S58, 2014

12. Kim BK, Kim DY, Park JY, Ahn SH, Chon CY, Kim JK, Paik YH, Lee KS, Park YN and Han KH: Validation of FIB-4 and comparison with other simple noninvasive indices for predicting liver fibrosis and cirrhosis in hepatitis B virus-infected patients. Liver Int 30: 546-553, 2010.

13. Nishikawa H, Takata R, Enomoto H, Yoh K, Kishino K, Shimono Y, Iwata Y, Hasegawa K, Nakano C, Nishimura T, et al: Proposal of a predictive model for advanced fibrosis containing Wisteria floribunda agglutinin-positive Mac-2-binding protein in chronic hepatitis C. Hepatol Res 47: E74-E84, 2017.

14. Hansen JF, Juul Nielsen M, Nyström K, Leeming DJ, Lagging M, Norkrans G, Brehm Christensen P and Karsdal M: PRO-C3: A new and more precise collagen marker for liver fibrosis in patients with chronic hepatitis C. Scand J Gastroenterol 53: 83-87, 2018.

15. Luo Y, Oseini A, Gagnon R, Charles ED, Sidik K, Vincent R, Collen R, Idowu M, Contos MJ, Mirshahi F, et al: An Evaluation of the collagen fragments related to fibrogenesis and fibrolysis in nonalcoholic steatohepatitis. Sci Rep 8: 12414, 2018.

16. Yoshiji H, Noguchi R and Fukui H: Combined effect of an ACE inhibitor, perindopril, and interferon on liver fibrosis markers in patients with chronic hepatitis C. J Gastroenterol 40: 215-216, 2005.

17. Casals G, Fernández-Varo G, Melgar-Lesmes P, Marfà S, Reichenbach V, Morales-Ruiz M and Jiménez W: Factors involved in extracellular matrix turnover in human derived cardiomyocytes. Cell Physiol Biochem 32: 1125-1136, 2013.

18. Saw S, Zhao H, Tan P, Saw B and Sethi S: Evaluation of the automated ADVIA centaur ${ }^{\circledR}$ XP syphilis assay for serological testing. Diagn Microbiol Infect Dis 88: 7-11, 2017.

19. Hogemann B, Voss B, Pott G, Rauterberg J and Gerlach U: 7 S collagen: A method for the measurement of serum concentrations in man. Clin Chim Acta 144: 1-10, 1984.

20. Jekarl DW, Choi H, Lee S, Kwon JH, Lee SW, Yu H, Kim M, Kim Y, Sung PS and Yoon SK: Diagnosis of liver fibrosis with wisteria floribunda agglutinin-positive Mac-2 binding protein (WFA-M2BP) among chronic hepatitis B patients. Ann Lab Med 38: 348-354, 2018 .

21. Nielsen MJ, Nedergaard AF, Sun S, Veidal SS, Larsen L, Zheng Q, Suetta C, Henriksen K, Christiansen C, Karsdal MA and Leeming DJ: The neo-epitope specific PRO-C3 ELISA measures true formation of type III collagen associated with liver and muscle parameters. Am J Transl Res 5: 303-315, 2013.

22. Noguchi R, Kaji K, Namisaki T, Moriya K, Kitade M, Takeda K, Kawaratani H, Okura Y, Aihara Y, Furukawa M, et al: Serum angiotensin-converting enzyme level for evaluating significant fibrosis in chronic hepatitis B. World J Gastroenterol 23: 6705-6714, 2017

23. Treatment with ursodeoxycholic acid in clinical hepatology. Proceedings of a workshop. Goteborg, Sweden, 3-4 February 1994. Scand J Gastroenterol Suppl 204: 1-72, 1994.
24. Intraobserver and interobserver variations in liver biopsy interpretation in patients with chronic hepatitis $\mathrm{C}$. The French METAVIR Cooperative Study Group. Hepatology 20: 15-20, 1994.

25. Rossi E, Adams LA, Bulsara M and Jeffrey GP: Assessing liver fibrosis with serum marker models. Clin Biochem Rev 28: 3-10, 2007.

26. Yamada N and Mizuta K: Advanced assessment of serum Mac-2 binding protein glycosylation isomer in patients with biliary atresia. J Gastroenterol 54: 204-205, 2019.

27. Shirabe K, Bekki Y, Gantumur D, Araki K, Ishii N, Kuno A, Narimatsu $\mathrm{H}$ and Mizokami M: Mac-2 binding protein glycan isomer (M2BPGi) is a new serum biomarker for assessing liver fibrosis: More than a biomarker of liver fibrosis. J Gastroenterol 53: 819-826, 2018.

28. Bekki Y, Yoshizumi T, Shimoda S, Itoh S, Harimoto N,Ikegami T, Kuno A, Narimatsu H, Shirabe K and Maehara Y: Hepatic stellate cells secreting $\mathrm{WFA}^{+}-\mathrm{M} 2 \mathrm{BP}$ : Its role in biological interactions with Kupffer cells. J Gastroenterol Hepatol 32: 1387-1393, 2017.

29. Matsuura K, Aizawa N, Enomoto H, Nishiguchi S, Toyoda H, Kumada T, Iio E, Ito K, Ogawa S, Isogawa M, et al: Circulating let-7 levels in serum correlate with the severity of hepatic fibrosis in chronic hepatitis C. Open Forum Infect Dis 5: ofy268, 2018.

30. Ishii A, Nishikawa H,Enomoto H, Iwata Y, Kishino K, Shimono Y, Hasegawa K, Nakano C, Takata R, Nishimura T, et al: Clinical implications of serum Wisteria floribunda agglutinin-positive Mac-2-binding protein in treatment-naive chronic hepatitis B. Hepatol Res 47: 204-215, 2017.

31. Nishikawa H, Enomoto H, Iwata Y, Kishino K, Shimono Y, Hasegawa K, Nakano C, Takata R, Nishimura T, Yoh K, et al: Serum Wisteria floribunda agglutinin-positive Mac-2-binding protein for patients with chronic hepatitis B and C: A comparative study. J Viral Hepat 23: 977-984, 2016.

32. Miyaki E, Imamura M, Hiraga N, Murakami E, Kawaoka T, Tsuge $\mathrm{M}$, et al. Daclatasvir and asunaprevir treatment improves liver function parameters and reduces liver fibrosis markers in chronic hepatitis C patients. Hepatol Res. 2016;46(8):758-64.

33. Liu J, Hu HH, Lee MH, Korenaga M, Jen CL, Batrla-Utermann $\mathrm{R}$, et al. Serum Levels of M2BPGi as Short-Term Predictors of Hepatocellular Carcinoma in Untreated Chronic Hepatitis B Patients. Sci Rep. 2017;7(1):14352.

34. Nishimura T, Iijima H, Nishikawa H, Kondo R, Yano H, Kage M, Aoki T, Nakano C, Yuri Y, Ishii N, et al: Liver fibrosis markers as assessed by ultrasound elastography and serum samples: A large comparative study in hepatitis virus B and $\mathrm{C}$ liver diseases. Hepatol Res 49: 721-730, 2019.

35. Yasui Y, Kurosaki M, Komiyama Y, Takada H, Tamaki N, Watakabe K, Okada M, Wang W, Shimizu T, Kubota Y, et al: Wisteria floribunda agglutinin-positive Mac-2 binding protein predicts early occurrence of hepatocellular carcinoma after sustained virologic response by direct-acting antivirals for hepatitis C virus. Hepatol Res 48: 1131-1139, 2018.

36. Nakamura M, Kanda T, Jiang X, Haga Y, Takahashi K, Wu S, Yasui S, Nakamoto S and Yokosuka O: Serum microRNA-122 and Wisteria floribunda agglutinin-positive Mac-2 binding protein are useful tools for liquid biopsy of the patients with hepatitis B virus and advanced liver fibrosis. PLoS One 12: e0177302, 2017.

37. Mak LY, Wong DK, Cheung KS, Seto WK, Lai CL and Yuen MF: Role of serum M2BPGi levels on diagnosing significant liver fibrosis and cirrhosis in treated patients with chronic hepatitis B virus infection. Clin Transl Gastroenterol 9: 163, 2018.

This work is licensed under a Creative Commons Attribution-NonCommercial-NoDerivatives 4.0 International (CC BY-NC-ND 4.0) License. 